\title{
Recombinant Human Interleukin-11 Modulates Ion Transport and Mucosal Inflammation in the Small Intestine and Colon
}

\author{
Beverley Greenwood-Van Meerveld, Karl Tyler, and James C. Keith, Jr.
}

Basic Science Laboratories (BG-VM, KT), Oklahoma Foundation for Digestive Research, Oklahoma City, Oklahoma; and Genetics Institute (JCK), Andover, Massachusetts

\begin{abstract}
SUMMARY: Human recombinant interleukin 11 (rhlL-11) is a cytokine that suppresses the clinical signs of colitis in animal models of inflammatory bowel disease (IBD) and may be an effective therapeutic agent in the treatment of IBD. The objective of the current study was to investigate whether rhlL-11 was capable of reversing abnormalities in secretomotor function associated with gut inflammation. We investigated the effects of rhlL-11 on epithelial electrogenic ion transport in the jejunum and colon. Application of rhlL-11 (10 to $10,000 \mathrm{ng} / \mathrm{ml})$ at either the luminal or serosal side of mucosal sheets isolated from control rats induced a concentration-dependent reduction of transmural potential difference (PD) in the jejunum and decreased the short-circuit current (ISc), representative of active electrogenic transport, in the colon. To investigate the effect of rhlL-11 on an inflamed gut, we isolated jejunal and colonic tissue from HLA-B27 transgenic rats with active inflammation of the bowel that represents an animal model of IBD. In jejunum and colon isolated from HLA-B27 transgenic rats, basal electrogenic ion transport was significantly attenuated and, under these conditions, rhlL-11 caused no changes in either transmural PD or Isc. However, in HLA-B27 rats, pretreatment with subcutaneous doses of rhlL-11 suppressed the symptoms of diarrhea, normalized myeloperoxidase activity in the jejunum and colon and healed mucosal injury. In the jejunum from HLA-B27 rats, healing of the intestinal inflammatory response enhanced basal transmural PD and the rhIL-11-induced changes in mucosal ion transport resembled those seen in uninflamed controls. Conversely, in the colon, healing of the mucosa did not normalize basal active ion transport nor did it reverse the inhibition of rhIL-11-induced changes in colonic Isc. Our results suggest that endogenous IL-11 may act as a modulator of epithelial transport under physiologic conditions and may act as a potent anti-inflammatory cytokine during active intestinal inflammation. (Lab Invest 2000, 80:1269-1280).
\end{abstract}

$C$ rohn's disease and ulcerative colitis are chronic immune-mediated disorders described by the term inflammatory bowel disease (IBD) and characterized by an idiopathic inflammation of the gastrointestinal tract. The clinical symptoms of IBD include diarrhea, abdominal pain, fever, weight loss, severe fatigue, and increased risk of developing of colon cancer (Levin, 1992). Although the etiology of IBD remains uncertain, factors such as genetically transmitted immunologic susceptibility to infections or autoimmune reactions have been implicated (Calkins and Mendeloff, 1986). Regardless of the initiating event, the inflammatory injury of the intestinal epithelial mucosal tissue involves abnormal $T$ cell activation mediated by proinflammatory cytokines (MacDonald and Spencer, 1988; Targan et al, 1991). However, the histologic and immunologic evidence of chronic mu-

Received March 20, 2000.

The study was supported by a grant to BG-VM from Genetics Institute, Andover, Massachusetts.

Address reprint requests to: Dr. B. Greenwood-Van Meerveld, Basic Science Laboratories, Oklahoma Foundation for Digestive Research, Veteran's Administration Medical Center, Research Administration Room 151, 921 N.E. 13th Street, Oklahoma City, Oklahoma 73104. Fax.: (405) 2901719; E-mail:Beverley-Greenwood@ouhsc.edu cosal inflammation in IBD lack an equally strong functional counterpart because little is known about the steps leading from mucosal immune activation and tissue injury to impairment of epithelial barrier and transport function.

Previous studies have suggested that cytokines, in addition to their immunomodulating effect, may act directly to regulate gastrointestinal function including intestinal water and ion transport. Evidence in support of this notion is that anti-inflammatory cytokines, such as interleukin (IL)-10 and IL-4, enhance intestinal absorption of sodium and chloride and inhibit chloride secretion (Madsen et al, 1996, 1997; Zund et al, 1996), whereas pro-inflammatory cytokines, such as IL-1 and IL-3, stimulate chloride secretion in vitro (Chiossone et al, 1990; Theodorou et al, 1994). Previous research with IL-11 suggests that this cytokine may enhance absorption of water and electrolytes within the gastrointestinal tract. Castagliuolo et al (1997) demonstrated that IL-11 significantly inhibits the secretion of fluids in rat ileal loops mediated by $C$. difficile toxin. Moreover, in rats with IBD, IL-11 was found to normalize stool consistency (Keith et al, 1994). Additional studies showed that IL-11 also enhances the gastrointestinal absorption of iron in rats (Baynes et al, 1995). Although these findings suggest that IL-11 may have a 
direct effect on the neuro-epithelial apparatus regulating the mucosal transport of electrolytes and water, there is still no evidence to confirm the possible action of IL-11 as a direct mediator of changes in intestinal ion transport.

IL-11 is a hematopoietic growth factor cloned by Paul et al (1990) that has effects on multiple cell types both in vivo and in vitro (Leng and Elias, 1997; Quesniaux et al, 1993). Recently, the availability of recombinant human interleukin-11 (rhlL-11), a 177-amino acid protein expressed in Escherichia coli, has enabled the analysis of structure-function relationships (Czupryn et al, 1995). In the gastrointestinal tract, rhlL-11 has been demonstrated to have protective effects in murine models of ischemic bowel necrosis (Du et al, 1997) and in rodent models of gut inflammation (Albert et al, 1997; Keith et al, 1994; Orazi et al, 1996; Qiu et al, 1996; Sonis et al, 1995). In on-going clinical trials, rhIL-11 therapy seems to be beneficial in patients with IBD (Sands et al, 1999).

The objectives of this study were to investigate rhlL-11 effects on the net electrogenic transport of electrolytes across the intestinal mucosa and to determine the effects of treatment with rhlL-11 in the HLA-B27 rat model of spontaneous, chronic intestinal inflammation. The HLA-B27 rat model is genetically engineered to overexpress the human major histocompatibility class 1 allele B27 and $\beta_{2}$-microglobulin gene products, associated with the development of chronic inflammatory disease (Hammer et al, 1990). In previous studies, treatment of HLA-B27 transgenic rats with rhlL-11 decreased the clinical symptoms of colitis and lowered the histologic damage score in the colon (Keith et al, 1994; Peterson et al, 1998). In the present study, we investigated the effect of rhIL-11 on intestinal net electrogenic transport using both an in vitro model of isolated jejunal and colonic mucosal sheets and an in vivo treatment model where rhlL-11 was administered to HLA-B27 transgenic rats exhibiting the symptoms associated with spontaneous and long-term inflammation of the alimentary tract.

\section{Results}

\section{Direct Effect of rhlL-11 on Transmucosal Net Electrogenic Transport in Noninflamed Intestinal Mucosa}

After equilibration, the jejunal mucosal sheets isolated from nontransgenic rats had a basal potential difference (PD) of $-3.3 \pm 0.2 \mathrm{mV}$ and a basal short-circuit current (Isc) of $26 \pm 5 \mu \mathrm{A} / \mathrm{cm}^{2}$. Consequently, the mean value of basal electrical conductance of the tissue was $8 \mathrm{mS} / \mathrm{cm}^{2}$. Addition of rhlL-11 to the serosal bathing medium induced a significant decrease in transmural PD that was concentrationdependent with a threshold of $10 \mathrm{ng} / \mathrm{ml}$ (Fig. 1A). In contrast, rhlL-11 induced a small decrease in jejunal Isc that was not statistically significant from the changes induced by the vehicle (data not shown). A series of control experiments demonstrated that an inactive form of the rhIL-11 molecule or its vehicle $(1 \%$ BSA in $0.9 \%$ saline) had no effects on jejunal electrical responses. The involvement of intramural neurons in the effect of rhlL-11 was tested in the presence of tetrodotoxin (TTX), which blocks sodium channels and inhibits neuronal activity. TTX (1 $\mu \mathrm{M}$ in serosal bathing solution) did not affect basal transmural PD in the jejunum and had no significant influence on the decrease in PD induced by rhlL-11 (Fig. 1B).

In the colon of nontransgenic rats, the basal PD was $-2.7 \pm 0.5 \mathrm{mV}$ and the basal Isc was $36 \pm 6 \mu \mathrm{A} / \mathrm{cm}^{2}$ with a corresponding basal electrical conductance of $13 \mathrm{mS} / \mathrm{cm}^{2}$. The serosal application of rhlL-11 produced a decrease in both PD and Isc. However, whereas changes in PD were moderate (a maximal decrease of $0.6 \pm 0.3 \mathrm{mV}$ ), rhlL-11 application induced a steep concentration-dependent reduction of the basal Isc, with a threshold of $10 \mathrm{ng} / \mathrm{ml}$ of $\mathrm{rhlL}-11$. The inactive form of the rhlL-11 molecule, as well as its vehicle (1\% BSA in $0.9 \%$ saline), had no effects on colonic electrical responses (Fig. 2A). In contrast to the jejunum, in colonic mucosal sheets, TTX itself produced an immediate and sustained decrease in basal Isc (from $37 \pm 3$ to $28 \pm 4 \mu \mathrm{A} / \mathrm{cm}^{2}, p<0.05$ ). Subsequently, in these TTX-treated tissues, administration of rhlL-11, up to concentrations of 10,000 $\mathrm{ng} / \mathrm{ml}$, did not produce any change in Isc (Fig. 2B). When intestinal mucosal sheets from the same rats were pretreated with the vehicle used to make up TTX (citrate buffer, $\mathrm{pH} 4.3$ ), administration of rhlL-11 produced the expected concentration-dependent decrease in Isc (Fig. 2B).

\section{Time Course of rhIL-11 on the Net Electrogenic Transport in Noninflamed Intestinal Mucosa}

The time-course of the responses produced by the direct interaction of rhlL-11 with jejunal or colonic intestinal mucosa is illustrated in Table 1. The data demonstrate that the onset of changes in jejunal PD (change in basal PD $>0.1 \mathrm{mV}$ ) occurred within the first 30 seconds after adding rhlL-11 to the serosal bathing solution, whereas the onset of rhlL-11-induced change in colonic Isc (changes in basal Isc $>3$ $\mu \mathrm{A} / \mathrm{cm}^{2}$ ) occurred with a latency of 4 minutes. On average, the time for the rhlL-11-induced changes in jejunal PD or colonic Isc to reach maximum was approximately 30 minutes. Neither the latency nor the time required by a single concentration of rhlL-11 to achieve a maximal effect were concentration dependent. Although we attempted to measure the duration of the responses, we noted that after 60 minutes there was no obvious return to baseline in the transmural PD or Isc responses, therefore longer time periods were not examined. However, at the 60-minutes time point, the addition of a maximal response dose of acetylcholine $(1 \mu \mathrm{M})$ to the serosal bathing solution induced the expected rapid increase in PD or Isc, proving the viability of the mucosal tissue.

\section{Site of Action of rhlL-11 on the Net Electrogenic Transport in Noninflamed Intestinal Mucosa}

To determine whether there is a luminal site of action for rhlL-11, we performed a series of experiments 

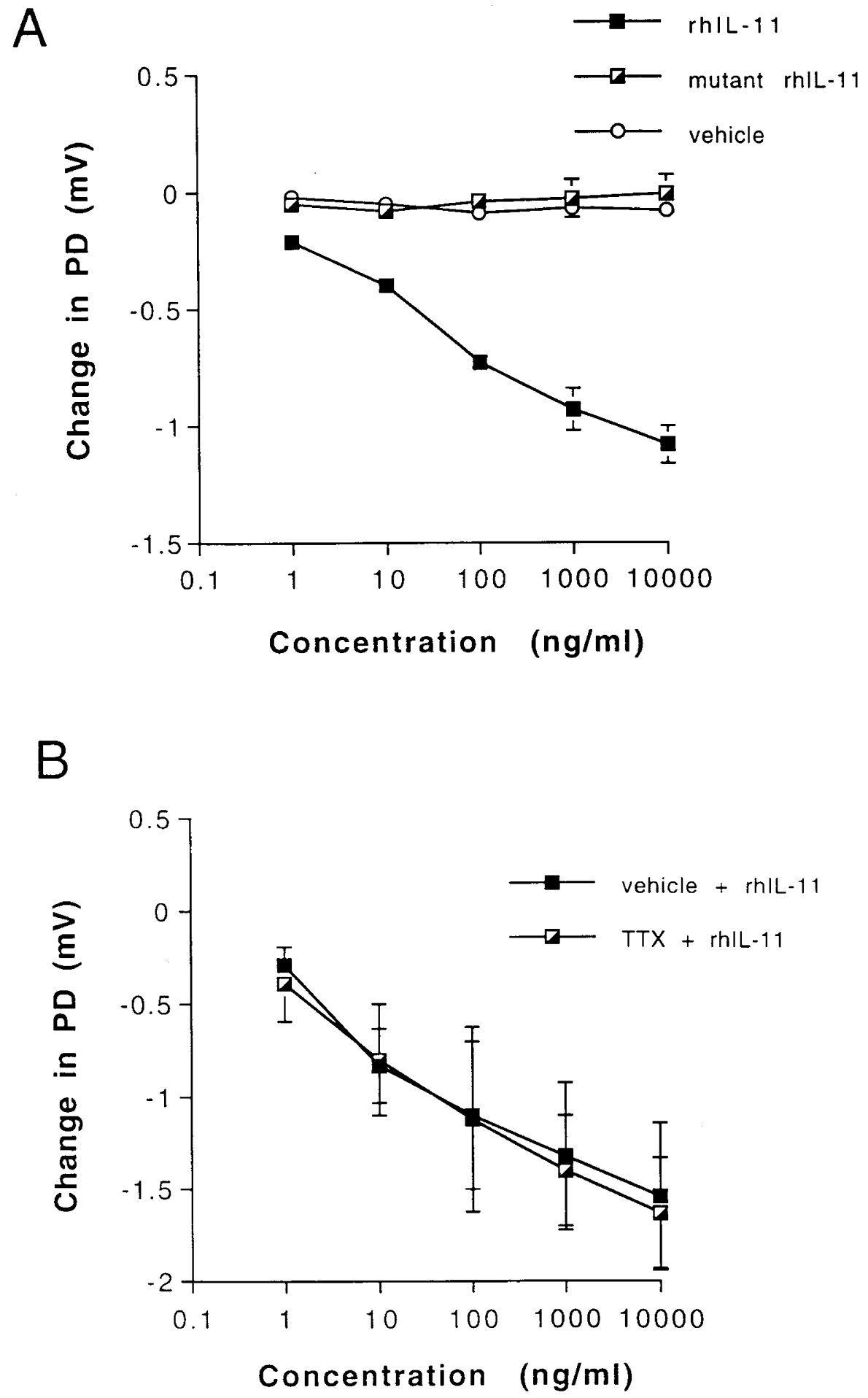

\section{Figure 1.}

Changes in transmural potential difference (PD) in isolated mucosal sheets from the jejunum of control rats (basal PD $=-3.2 \pm 0.2 \mathrm{mV}, n=7$ rats). Each data point represents the effects measured at their maximum (within 8 to 10 minutes) and expressed as mean \pm SEM from 6 to 12 experiments. ${ }^{\star} p<0.05$ vs vehicle control. A, Serosal application of increasing concentrations of human recombinant interleukin 11 (rhIL-11) causes a concentration-dependent reduction in basal PD, whereas an inactive rhIL-11 mutant molecule or the vehicle $(0.1 \%$ BSA in saline) has no significant effect. B, Effects of increasing-concentrations of rhIL-11 on jejunal $\mathrm{PD}$ in tissues pretreated with $1 \mu \mathrm{M}$ tetrodotoxin (TTX) or with corresponding volumes of its vehicle (citrate buffer, $\mathrm{pH} 4.3$ ) added to the serosal bathing solution 15 minutes before rhlL-11 addition.

where rhlL-11 was added to the mucosal bathing solution and the results were compared with those obtained from serosal application of rhlL-11. We found that addition of rhlL-11 to the mucosal bathing solution produced changes in jejunal transmural PD and colonic Isc that were virtually the same as these 
A
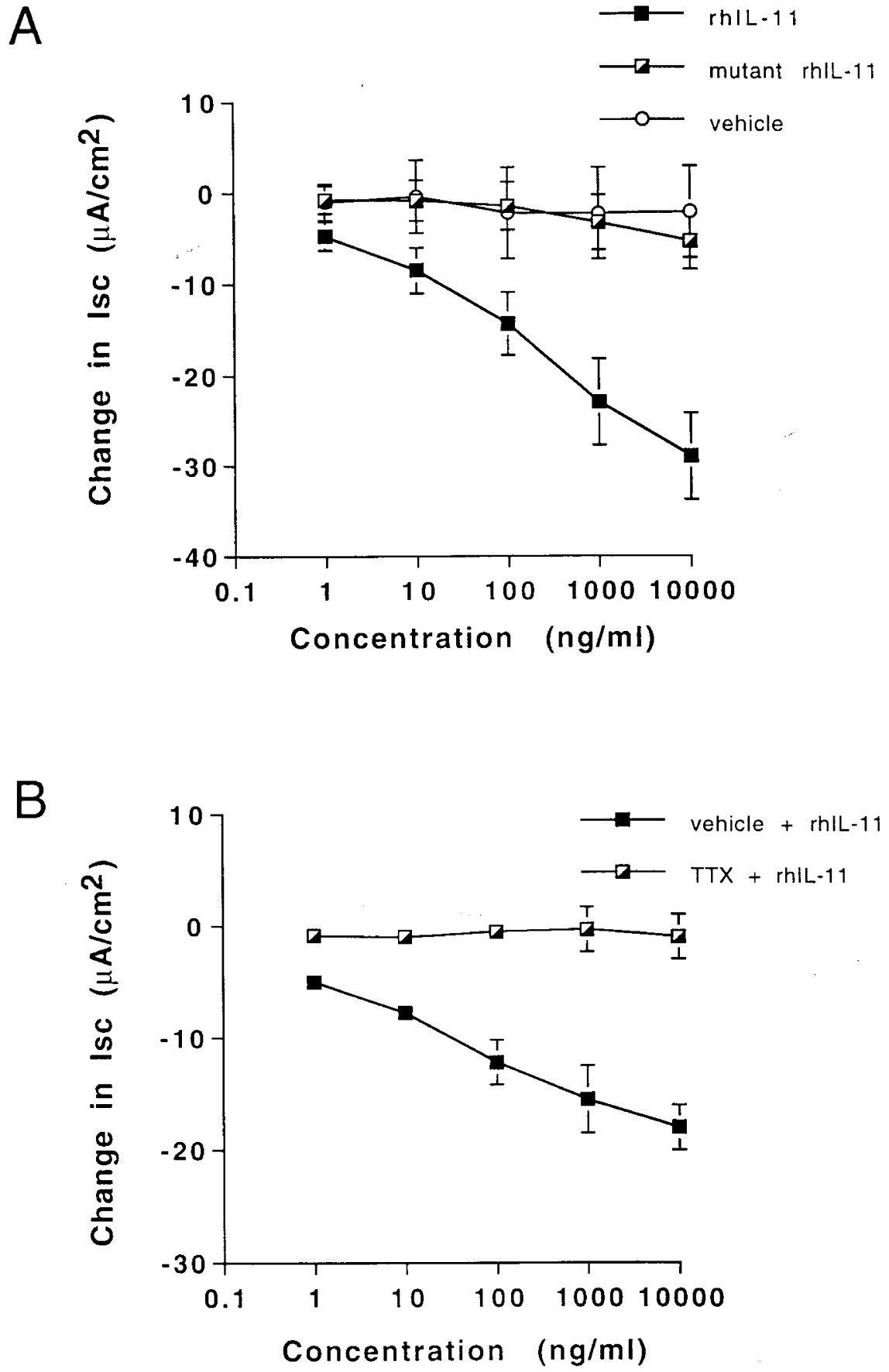

Figure 2.

Changes in transmucosal short-circuit current (Isc) in isolated mucosal sheets from the colon of control rats (basal Isc $=37 \pm 3 \mu \mathrm{A} / \mathrm{cm}^{2}, n=7 \mathrm{rats}$ ). Each data point represents the effects measured at their maximum (within 8 to 10 minutes) and expressed as mean \pm SEM from 6 to 9 experiments. ${ }^{*} p<0.05$ vs vehicle control. A, Serosal application of increasing concentrations of rhIL-11 causes a concentration-dependent reduction in basal Isc, whereas an inactive rhIL-11 mutant molecule or the vehicle (0.1\% BSA in saline) has no significant effect. B, Effects of increasing concentrations of rhIL-11 on colonic Isc in tissues pretreated with TTX (1 $\mu \mathrm{M})$ or with corresponding volumes of its vehicle (citrate buffer, $\mathrm{pH} 4.3$ ) added to the serosal bathing solution 15 minutes before rhIL-11 application.

produced by the serosal addition of rhIL-11. In the jejunum, after mucosal administration, the maximal decrease in transmural PD was $1.1 \pm 0.2 \mathrm{mV}(n=12)$ versus $1.1 \pm 0.3 \mathrm{mV}(n=9)$ after serosal administration. In the colon, the maximal decrease in Isc was $25 \pm 1.7 \mu \mathrm{A} / \mathrm{cm}^{2}(n=12)$ after mucosal administration versus $29 \pm 4.8 \mu \mathrm{A} / \mathrm{cm}^{2}(n=9)$ after serosal administration.

\section{Effect of Inflammation on rhlL-11-Induced Net Electrogenic Transport in the Intestinal Mucosa of HLA-B27 Transgenic Rats}

The HLA-B27 transgenic rats used in this study had developed a chronic inflammation of the gastrointestinal tract, manifested by the clinical signs of persistent diarrhea (Hammer et al, 1990). Intestinal inflam- 
Table 1. Latency of the Onset and Time to Reach Maximum for the Effects of Serosal Application of rhIL-11 on Jejunal PD and Colonic Isc in the Ussing Chamber

\begin{tabular}{lccc}
\hline $\begin{array}{c}\text { rhlL-11 } \\
(\mathrm{ng} / \mathrm{ml})\end{array}$ & $\begin{array}{c}\text { Response Latency } \\
(\mathrm{min})\end{array}$ & $\begin{array}{c}\text { Time to Max. } \\
(\mathrm{min})\end{array}$ & $\mathrm{n}$ \\
\hline $\begin{array}{c}\text { Jejunum (PD) } \\
10\end{array}$ & $0.58 \pm 0.08$ & $31.3 \pm 1.4$ & 6 \\
100 & $0.49 \pm 0.02$ & $34.3 \pm 1.8$ & 6 \\
1000 & $0.54 \pm 0.03$ & $38.8 \pm 2.1$ & 6 \\
10,000 & $0.51 \pm 0.03$ & $38.1 \pm 1.9$ & 6 \\
Colon (Isc) & & & \\
10 & $3.97 \pm 0.28$ & $30.0 \pm 3.8$ & 6 \\
100 & $4.30 \pm 0.68$ & $27.2 \pm 1.7$ & 6 \\
1000 & $4.07 \pm 0.15$ & $32.4 \pm 1.3$ & 6 \\
10,000 & $4.07 \pm 0.13$ & $31.8 \pm 1.0$ & 6 \\
\hline
\end{tabular}

Values are given as mean $\pm \mathrm{SEM}$.

mation in these animals was confirmed by the significant elevation of myeloperoxidase (MPO) activity in both the jejunum $(90 \pm 15 \mathrm{ng} / \mathrm{g}$ tissue in HLA-B27 rats versus $27 \pm 13 \mathrm{ng} / \mathrm{g}$ tissue in controls, $p<0.01)$ and colon (189 $\pm 28 \mathrm{ng} / \mathrm{g}$ tissue in HLA-B27 rats versus $37 \pm 5 \mathrm{ng} / \mathrm{g}$ tissue in controls, $p<0.01$ ). Additionally, the basal transmural PD and Isc measured in isolated jejunal and colonic mucosal sheets from HLA-B27 rats were significantly lower than in control rats. The basal transmural $P D$ in the jejunum of HLA-B27 was $-1.6 \pm 0.3 \mathrm{mV}$ (12 mucosal sheets from three rats) and was significantly different $(p<0.001)$ from the control value of $-3.0 \pm 0.2 \mathrm{mV}$ (12 mucosal sheets from three rats) in healthy nontransgenic rats. Similarly, the basal Isc in the jejunum of $19 \pm 3$ $\mu \mathrm{A} / \mathrm{cm}^{2}$ in HLA-B27 rats was reduced compared with the $26 \pm 5 \mu \mathrm{A} / \mathrm{cm}^{2}$ measured in controls. Furthermore, an increase in the mean basal conductance of the jejunum was observed in the HLA-B27 rats (12 $\mathrm{mS} / \mathrm{cm}^{2}$ ) compared with nontransgenic controls (8 $\mathrm{mS} / \mathrm{cm}^{2}$ ). In the colon from HLA-B27 rats, basal PD and Isc were both significantly reduced $(\mathrm{PD}=-1.6 \pm$ $0.3 \mathrm{mV}$; Isc $=17 \pm 3.5 \mu \mathrm{A} / \mathrm{cm}^{2}, 12$ mucosal sheets from three rats) compared with nontransgenic controls $\left(\mathrm{PD}=-2.7 \pm 0.5 \mathrm{mV}\right.$; Isc $=33 \pm 2.3 \mu \mathrm{A} / \mathrm{cm}^{2} ; 12$ mucosal sheets from three rats, $p<0.001)$. However, tissue conductance values were similar in HLA-B27 rats $\left(11 \mathrm{mS} / \mathrm{cm}^{2}\right)$ and healthy nontransgenic controls $\left(13 \mathrm{mS} / \mathrm{cm}^{2}\right)$. In isolated sheets of jejunum and colon harvested from HLA-B27 rats, we examined the direct effect of rhlL-11. The decreases in jejunal transmural PD and the increases in colonic Isc induced by rhIL-11 in healthy nontransgenic rats were absent or significantly attenuated in tissue from HLA-B27 transgenic rats (Fig. 3, A and $B$ ).

\section{Effect of rhlL-11 Treatment of HLA-B27 Transgenic Rats on Intestinal Inflammation and rhlL-11-Induced Net Electrogenic Transport}

In a separate series of experiments, HLA-B27 transgenic rats received rhIL-11 (33 $\mu \mathrm{g} / \mathrm{kg}$ subcutaneously
A

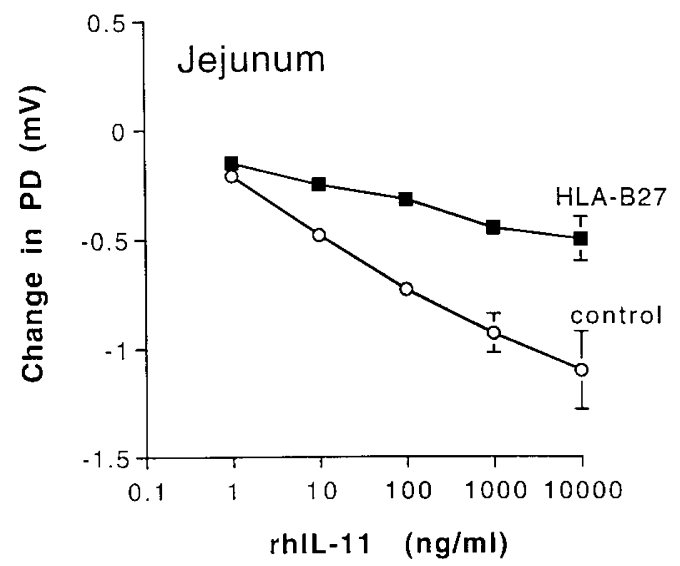

B

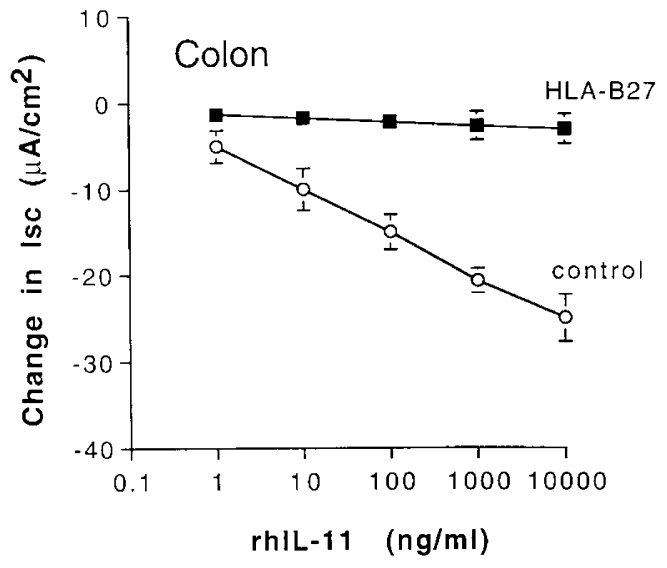

Figure 3.

Effects of increasing concentrations of rhIL-11 on jejunal PD (basal PD = $-3.0 \pm 0.2 \mathrm{mV}$ ) $(\mathrm{A})$ or colonic Isc (basal Isc $=33 \pm 2 \mu \mathrm{A} / \mathrm{cm} 2)(\mathrm{B})$ in mucosal sheets isolated from control rats ( $n=7$ rats) and naïve HLA-B27 transgenic rats ( $n=7$ rats). Data are mean \pm SEM from 8 to 10 experiments for each group.

(sc) on alternate days for either 1 or 2 weeks. Treatment with rhlL-11 showed an improvement of the clinical symptoms of IBD, normalizing stool consistency from diarrhea and loose stools to normal stool pellets. Whereas an improvement of stool character was evident after the first injection of rhlL-11, a maximal effect was obtained during the second week of treatment (Fig. 4). Moreover, after the 1 or 2 weeks of treatment with rhlL-11, the histologic damage of intestinal mucosa in HLA-B27 rats improved in a time-dependent fashion in both the jejunum and colon (Table 2, Fig. 5). These results closely correlated with the reduction in activity of the inflammatory marker MPO (Figs. 6A and 7A). Treatment of HLA-B27 transgenic rats with rhlL-11 resulted in a statistically significant increase in the basal PD across the jejunum $(p<$ 0.05 ) after 2 weeks of treatment (Fig. 6B). Furthermore, the mean value of electrical conductance across the jejunum after 1 week of rhlL-11 treatment $(6$ $\mathrm{mS} / \mathrm{cm}^{2}$ versus $12 \mathrm{mS} / \mathrm{cm}^{2}$ in naive HLA-B27 rats) was sustained after the 2 weeks of treatment. The basal jejunal Isc of $17 \pm 2 \mu \mathrm{A} / \mathrm{cm}^{2}$ in HLA-B27 rats treated with rhIL-11 for 2 weeks resembled that seen 


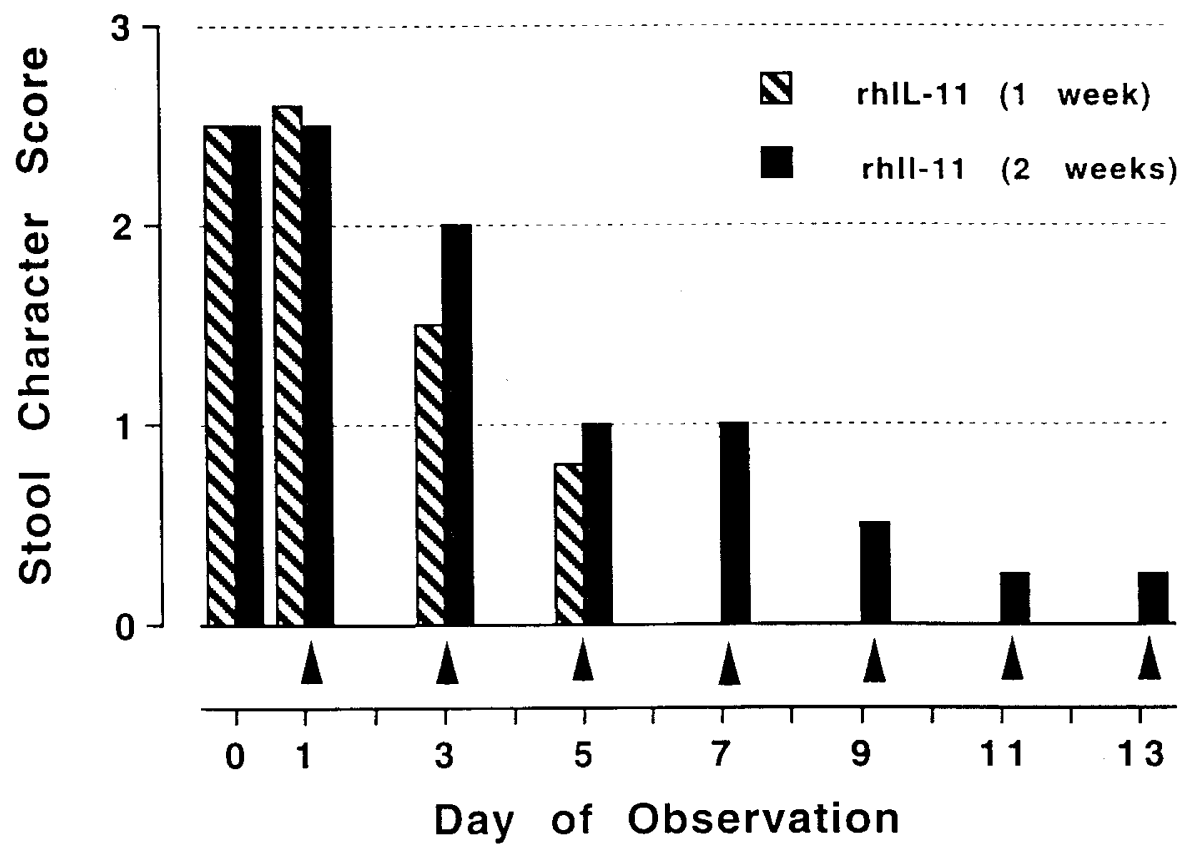

Figure 4.

Stool character in HLA-B27 rats receiving $33 \mu \mathrm{g} / \mathrm{kg}$ of rhlL-11 subcutaneously (sc) on alternate days for 1 or 2 weeks. Stool consistency was scored as follows: 0 , normal with formed stool pellets; 1 , soft with formed stool pellets; 2, loose, no formation of stool pellets; or 3 , watery diarrhea. Stools were scored the day before the beginning of rhIL-11 treatment (Day 0 ) and every other day during the treatment period. Data are mean each of the groups $(n=4$ rats); arrows indicate the days on which rhlL-11 was administered and stool consistency was scored.

Table 2. Histological Lesion Scores in HLA-B27 Transgenic Rats Treated with rhlL-11 (33 $\mu \mathrm{g} / \mathrm{kg}$ s.c. on Alternate Days for One or Two Weeks)

\begin{tabular}{|c|c|c|c|c|c|}
\hline & Ulceration & Inflammation & Lesion Depth & Fibrosis & Total \\
\hline \multicolumn{6}{|l|}{ Jejunum } \\
\hline Naïve & $1.9 \pm 0.3$ & $3.0 \pm 0.0$ & $1.9 \pm 0.7$ & $0.5 \pm 0.6$ & $7.3 \pm 1.3$ \\
\hline \multicolumn{6}{|l|}{ Treated } \\
\hline 1 week & $0.5 \pm 0.7^{\star}$ & $1.3 \pm 0.8^{*}$ & $0.5 \pm 0.7^{\star}$ & $0.3 \pm 0.5$ & $2.5 \pm 1.6^{*}$ \\
\hline 2 weeks & $0.2 \pm 0.4^{*}$ & $0.7 \pm 0.8^{*}$ & $0.2 \pm 0.4^{*}$ & $0.4 \pm 0.5$ & $1.4 \pm 1.0^{*}$ \\
\hline \multicolumn{6}{|l|}{ Colon } \\
\hline \multicolumn{6}{|l|}{ Treated } \\
\hline 1 week & $0.3 \pm 0.5^{\star}$ & $1.6 \pm 0.5^{\star}$ & $0.3 \pm 0.5^{\star}$ & $0.3 \pm 0.5^{\star}$ & $2.4 \pm 0.3^{*}$ \\
\hline 2 weeks & $0.2 \pm 0.4^{*}$ & $1.0 \pm 0.6^{*}$ & $0.2 \pm 0.4^{*}$ & $0.3 \pm 0.5^{*}$ & $1.6 \pm 0.9^{*}$ \\
\hline
\end{tabular}

Values are mean \pm SEM $(n=6$ for each group)

* Denotes lower than untreated $(p<0.05)$.

in naive HLA-B27 rats $\left(19 \pm 3 \mu \mathrm{A} / \mathrm{cm}^{2}\right)$. Recovery of the jejunal mucosa in HLA-B27 transgenic rats was associated with an increase in the rhlL-11-induced change in PD that resembled control rats, however the normalization of the PD response due to direct stimulation with rhIL-11 was not significantly different from naive rats and was not sustained during the second week of rhlL-11 pretreatment. (Fig. 6C). In the colon, histologic improvement and the significant decrease in MPO activity (Fig. 7A) was associated with a normalization of basal PD in HLA-B27 rats pretreated with rhlL-11. Colonic basal PD in mucosal sheets from HLA-B27 rats given rhlL-11 for 2 weeks $(-2.4 \pm 0.5$ $\mathrm{mV}$ ) did not differ significantly from the respective value in mucosal sheets isolated from control rats $(-2.7 \pm 0.5 \mathrm{mV})$. Concurrently, the mean electrical conductance was slightly lower $\left(9 \mathrm{mS} / \mathrm{cm}^{2}\right)$ in HLA$\mathrm{B} 27$ rats treated with rhlL-11 for 2 weeks compared with both control $\left(13 \mathrm{mS} / \mathrm{cm}^{2}\right)$ or naive HLA-B27 rats $\left(11 \mathrm{mS} / \mathrm{cm}^{2}\right)$. However, in the colon, despite the histologic improvement and the significant decrease in MPO activity caused by rhlL-11 treatment of HLA-B27 rats, neither the basal Isc (Fig. 7B) nor the Isc response to direct stimulation with rhlL-11 were significantly improved (Fig. 7C).

\section{Discussion}

In this study, the effects of rhlL-11 on mucosal ion transport in rat jejunum and colon were investigated. Our results indicate that in addition to its immunomodulating activity, rhlL-11 may directly regulate in- 

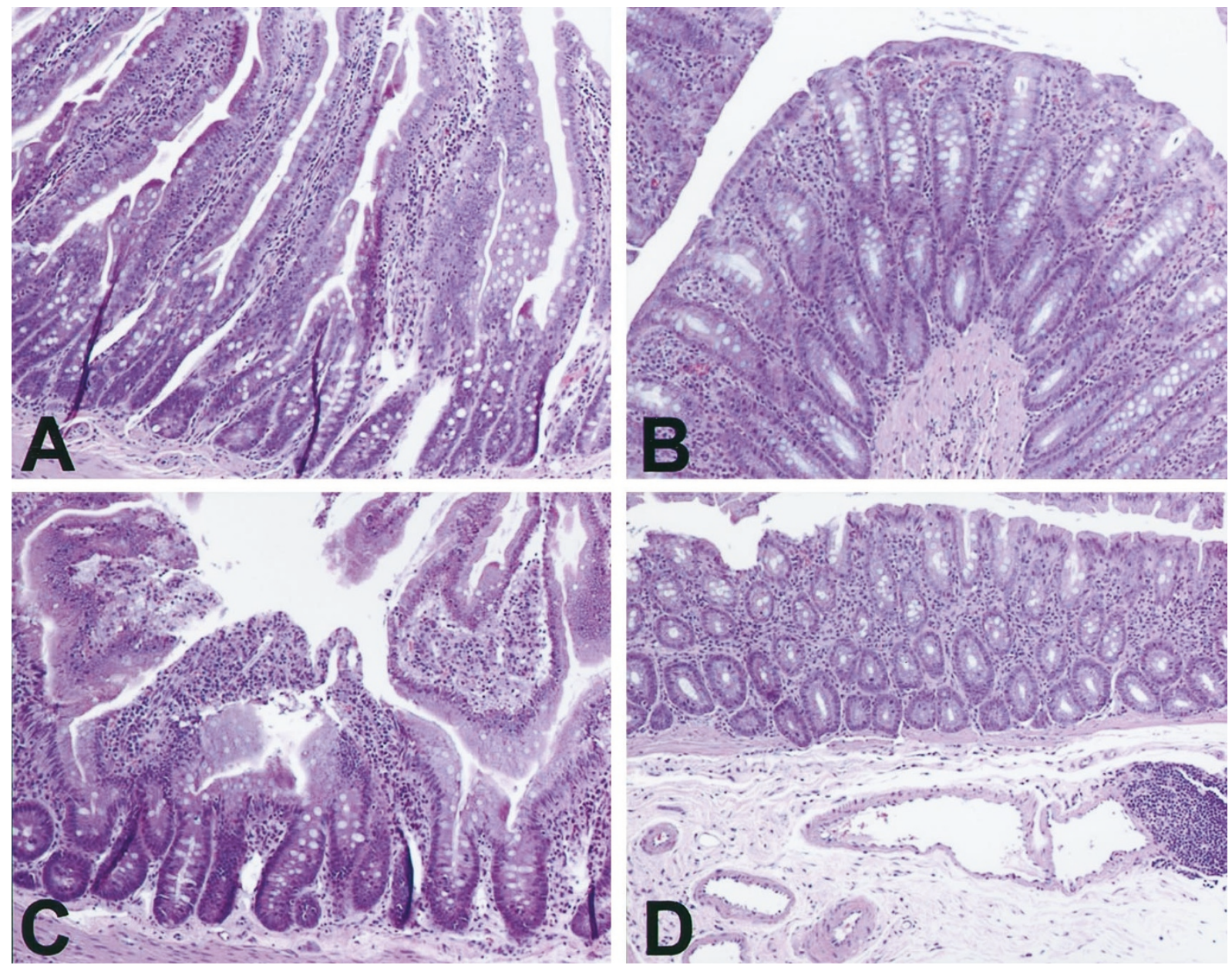

\section{Figure 5.}

Photomicrographs of jejunum (A) and colon (B) of rats receiving rhIL-11 for two weeks exhibit fewer inflammatory infiltrates and an intact luminal border. In contrast, in vehicle-treated animals, note the marked inflammatory infiltrates and shortened villi in the jejunum $(C)$ and the uneven luminal border and submucosal inflammatory infiltrates in the colon (D). Hematoxylin and eosin stain. Original magnification, $\times 100$.

testinal mucosal transport. In the absence of inflammation, rhlL-11 alters ion transport across the rat small intestine and colon in a concentrationdependent manner. Under resting conditions, the jejunum maintains a basal transmural PD with the mucosal side negative compared with the serosal. This negative PD is, for the most part, generated through the electrogenic absorption of sodium ions and active electrogenic secretion of chloride ions into the lumen. The addition of rhlL-11 to either the serosal or luminal side of the jejunum decreased the basal transmural $\mathrm{PD}$, causing concentration-dependent changes of the PD towards less negative values in the lumen. The changes induced by rhlL-11 also involved a small decrease in Isc, which is an electrical marker of active energy-dependent ion transport in the absence of or against electrochemical gradients. Taken together, our findings suggest that in the rat jejunum a reduction in transmural PD produced by rhIL-11 is not the result of any major change in active transport processes and it is more likely that rhlL-11 alters transmural PD by changing passive transport processes. On the other hand, in the colon, the mechanism by which rhlL-11 alters ion transport seems to be different from that observed in the jejunum. Similar to the jejunum, the colon generates a lumen-negative transmural PD that is the net effect of both active and passive transport across the "tighter" colonic epithelium. However, in colonic mucosal sheets from nontransgenic control rats, rhlL-11 caused only a small decrease in transmural PD in the presence of a significant decrease in Isc. This finding supports the notion that rhIL-11 induces changes in active ion transport processes without significantly changing the passive transport of electrolytes across the colonic mucosa. Although relevant, this assumption should be regarded cautiously because the in vitro technique used in our study accounts only for the electrogenic transport of electrolytes and does not reflect electroneutral processes of transepithelial ion exchange. The existence of coupled $\mathrm{Na}^{+}$and $\mathrm{Cl}^{-}$absorption has been well documented in the colon (Binder et al, 1987; Sellin and Desoignie, 1984). It seems most likely that coupled $\mathrm{Na}^{+}$and $\mathrm{Cl}^{-}$absorption occurs via a series of active transport carrier proteins including a $\mathrm{Na} / \mathrm{H}$ exchanger and $\mathrm{a} \mathrm{Cl} / \mathrm{HCO}_{3}^{-}$exchanger. The data from the present study do not define whether rhIL-11 is capable of regulating that particular type of transepithelial trans- 

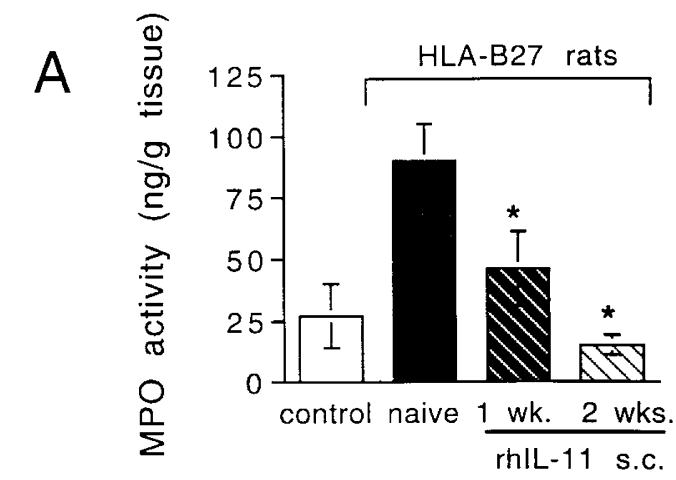

B
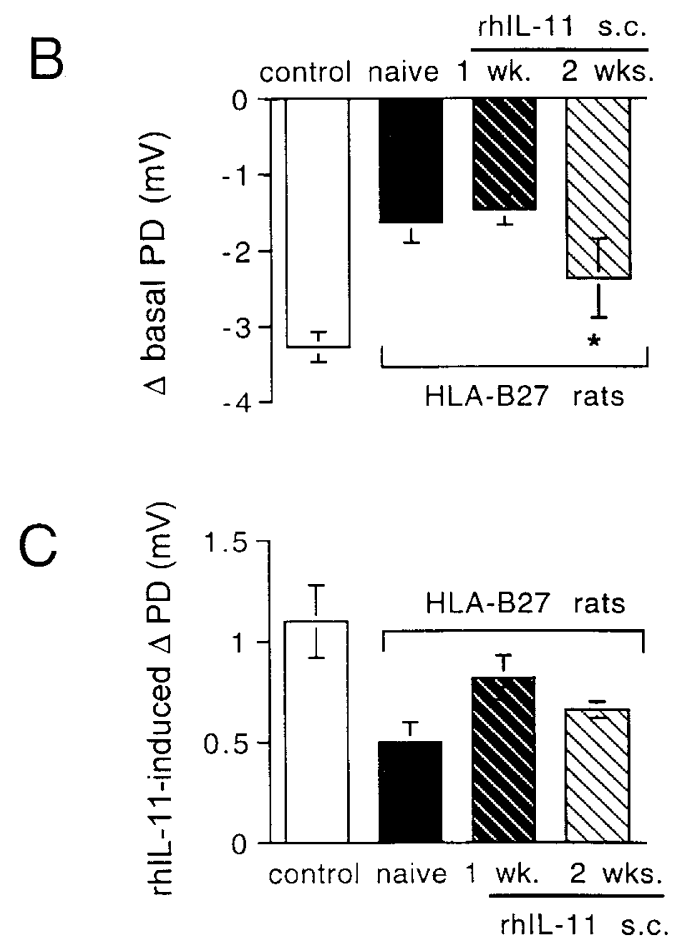

Figure 6.

Myeloperoxidase (MPO) activity (A), basal values of transmural PD (B), and maximal changes in basal PD induced by serosal application of rhIL-11 (C) in the jejunum of naïve HLA-B27 transgenic rats (filled black bars) and HLA-B27 rats treated with rhlL-11 (33 $\mu \mathrm{g} / \mathrm{kg} \mathrm{sc}$ at 48 hours intervals) during 1 or 2 weeks (hatched bars). Respective values obtained in jejunal tissue from control rats are also presented (open bars). Data for MPO activity are mean $\pm \mathrm{SE}$ calculated for 7 control or naive HLA-B27 rats and 4 HLA-B27 rats in each of the rhIL-11-treated groups. Data for PD are mean \pm SE from 8 to 12 experiments for each group. ${ }^{*} p<0.05$ vs naïve HLA-B27 transgenic rats.

port; this question remains to be answered by a different methodologic approach. Nevertheless, in the present experiments, the directions of changes in basal PD and Isc observed in the jejunum and colon in response to rhlL-11 are suggestive of a proabsorptive effect (Chang et al, 1982; Greenwood et al, 1987). Previous studies showing that rhIL-11 promotes the gastrointestinal absorption of iron in rats (Baynes et al 1995) and inhibits C. difficile toxin-mediated fluid secretion in ileal loops from the rat (Castagliuolo et al 1997) also support our claim that rhIL-11 alters mu-
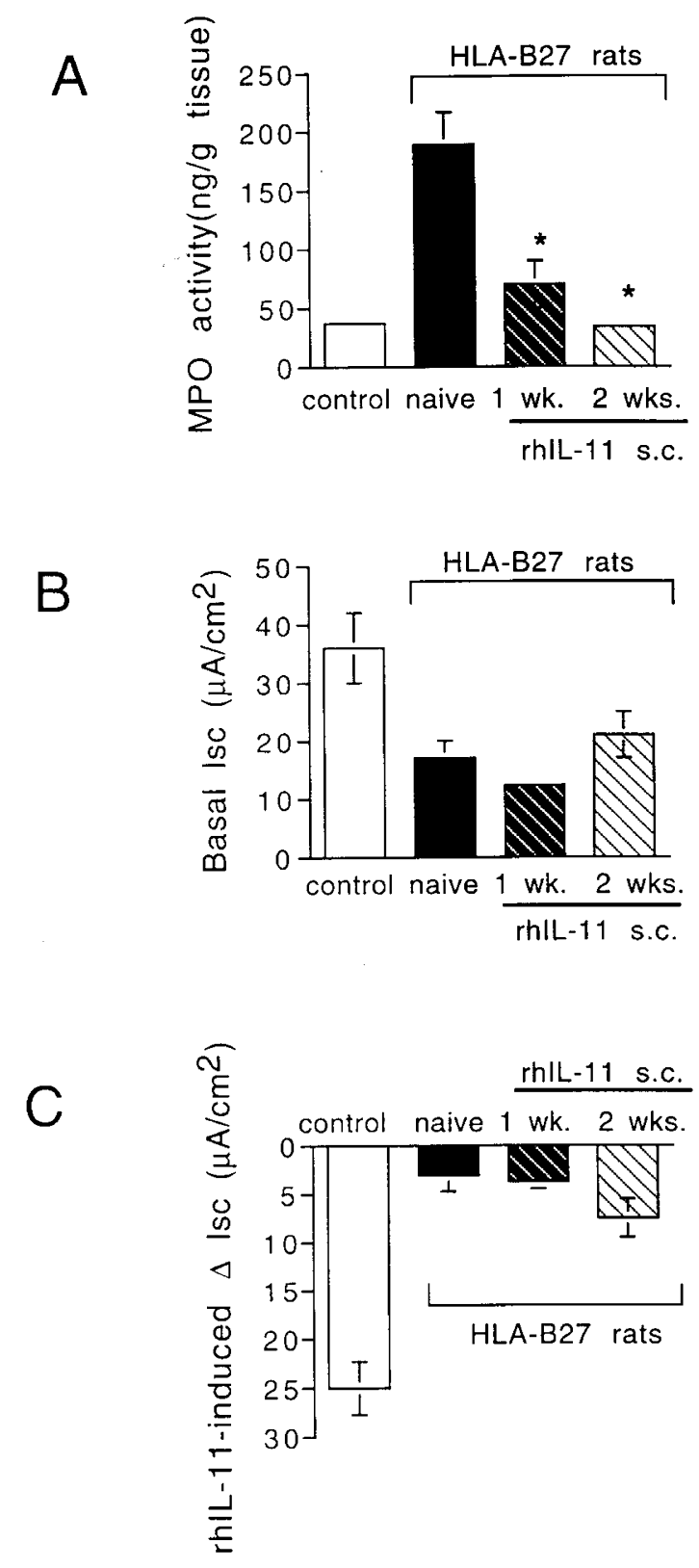

Figure 7.

Myeloperoxidase (MPO) activity (A), basal values of Isc (B), and maximal changes in Isc induced by serosal application of rhIL-11 (C) in the colon isolated from naïve HLA-B27 transgenic rats (filled black bars) and HLA-B27 rats treated with rhIL-11 (33 $\mu \mathrm{g} / \mathrm{kg}$ sc at 48 hours intervals) during 1 or 2 weeks (hatched bars). Respective values obtained in jejunal tissue from control rats are also presented (open bars). Data for MPO activity are mean \pm SE from 7 control or naive HLA-B27 rats and 4 HLA-B27 rats in each of the rhIL-11-treated groups. ISC data are mean \pm SE from 8 to 9 experiments for each group. * $p<0.05$ vs naïve HLA-B27 transgenic rats.

cosal transport in the direction of enhanced net intestinal absorption. Moreover, recent studies in rats with small bowel resection provided direct evidence that rhIL-11 enhances small intestinal absorptive function and mucosal mass (Alavi et al, 2000).

To further characterize the effect of rhlL-11 on ion transport, we examined the time course of electrical responses to rhIL-11 and discovered that time parameters differed significantly between the jejunum and 
colon. In the jejunum, there was a rapidly occurring increase in PD (within 30 seconds), whereas up to 4 minutes were required before there was a change in Isc in the colon. The reason for these differences is unclear, however it is evident that they are related to divergent mechanisms by which rhlL-11 causes changes in the passive ion transport in the jejunum or the active ion transport in the colon. The rapid onset of the jejunal PD response suggests a direct receptoroperated mechanism. The longer time period required for a change in Isc in the colon could involve an immune-mediated effect that is associated with a secondary release of a cytokine or neurotransmitter that acts to decrease basal Isc. In both regions of the intestinal tract, the effects of $\mathrm{rhlL}-11$ are likely to involve a specific receptor-mediated mechanism, because no effects were found in experiments using a rhlL-11 mutant molecule that has a 25-fold lower binding affinity for the high affinity chain of the rhlL-11 receptor. rhlL-11 has a prolonged duration of action on jejunal and colonic mucosal ion transport, because there was a 30 minutes lag for each concentration to achieve a maximal effect in both the jejunum and colon and the responses did not return to basal within the 60 -minute recording period. We have shown that rhIL-11-induced changes in the jejunal mucosal ion transport in the Ussing chamber are unlikely to involve neuronal mechanisms because the pretreatment of the tissue with the neurotoxin TTX was without effect on the rhlL-11-induced electrical response. Micromolar concentrations of TTX are known to block neuronal sodium channels (Kao, 1986), causing the inhibition of neurally mediated changes in intestinal epithelial transport (Perdue and Davison, 1986). In contrast, in the colon, our findings proved that TTX abolishes the decrease in active ion transport induced by rhlL-11, indicating that the functional integrity of neuro-effector junctions is required for realization of the rhlL-11mediated effect in the colon.

To address the potential local versus systemic site of action for rhIL-11, we performed experiments where concentration-response effects of either serosal or mucosal administration of rhlL-11 were investigated. The observations from this series of experiments demonstrated that rhIL-11 changes transmural PD in the jejunum and Isc in the colon after either serosal or mucosal administration, implying that rhIL-11 receptors may be located on both the apical and basolateral surfaces of the enterocyte. In support of a dual systemic and luminal site of action, studies by Albert et al (1997) showed that enema delivery of rhIL-11 is effective in both the HLA-B27 rat and the acetic acid model of acute colitis. Our data also suggest that rhIL-11 can affect intestinal function when administered to either the serosal or mucosal side of the tissue. Extrapolating our findings to an in vivo situation, we could postulate that administration of rhIL-11 possesses the capability of enhancing intestinal absorption and promoting antiinflammatory effects after either systemic or luminal administration. This observation is important because it provides preclinical data to support development of an enema formulation of rhlL-11 that can be used in patients with ulcerative colitis or in patients with Crohn's disease restricted to the colon.

To further understand the effects of rhIL-11 on ion transport under pathologic conditions, we investigated the effect of rhlL-11 in genetically engineered HLA-B27 transgenic rats with chronic, spontaneously induced inflammation of the gastrointestinal tract. In these rats, the magnitudes of the changes in jejunal transmural PD or in colonic Isc induced by rhIL-11 were significantly attenuated or completely abolished compared with those seen in normal rats. These findings demonstrate that mucosal inflammation decreased the rhlL-11 responsiveness of the small intestine and colon, suggesting that the action of endogenous IL-11 is confined to the uninflamed status of the tissue and may thus play a role in the maintenance of normal epithelial function.

Our observations that the basal transmural PD in HLA-B27 rats was less negative than in control rats and that basal tissue conductance was increased, support the notion that inflamed tissue may have increased permeability and therefore may be unable to generate large potential differences. To more completely understand the role of IL-11 in intestinal inflammation, we examined the in vitro effect of rhlL-11 in mucosal sheets isolated from HLA-B27 rats where the inflammatory disease has been reduced by in vivo pretreatment with rhlL-11 (Keith et al 1994; Peterson et al, 1998). Although basal PD and tissue conductance showed recovery in tissues from rhlL-11-pretreated HLA-B27 transgenic rats, the basal Isc did not show a significant recovery. Because inhibition of MPO activity and lowering of the histologic lesion score was achieved by 1 and 2 week treatments with rhlL-11 in both the jejunum and colon, it is possible that, unlike the structural restitution of the mucosa, the recovery of basal active electrolyte transport function in both the jejunum and colon requires a longer period of treatment with rhIL-11.

In the jejunum, the changes in ion transport measured electrically as changes in transmural PD produced by the local application of rhlL-11 to the serosal bathing solution were greater in HLA-B27 rats pretreated with rhlL-11 compared with untreated HLAB27 rats and more closely resembled the electrical responses observed in control rats. In the colon from HLA-B27 rats pretreated with rhlL-11, healing of the colonic inflammatory response did not reverse the complete lack of effect of rhlL-11 on Isc. This finding is in agreement with recent results obtained in a rat model of trinitrobenzene sulfonic acid-induced colitis, indicating a prolonged down-regulation of secretory responses to inflammatory mediators that persists after resolution of mucosal inflammation (Asfaha et al, 1999). Additionally, despite the lack of effect of rhIL-11 treatment of HLA-B27 rats on jejunal and colonic electrical epithelial responses to rhIL-11, we observed a significant improvement of the stool character. Although the exact mechanism of this effect is unclear, it is evident that healing of mucosal injury per se may have a beneficial effect improving paracellular permeability. 
In conclusion, our data demonstrate that local treatment with rhlL-11 may alter electrogenic ion transport across the small intestine and colon. The ion transport processes induced by rhIL-11 are severely attenuated by an inflammatory insult. Although systemic treatment of HLA-B27 transgenic rats with rhlL-11 is capable of healing the inflammatory injury in both the small and large intestine and reversing abnormalities in basal passive ion fluxes, active ion transport remains abnormal. Furthermore, the reversal of rhlL-11induced abnormalities in passive ion fluxes in the jejunum is incomplete and the active ion transport induced by rhlL-11 in the colon remains abnormal. Taken together, our results indicate that endogenous $\mathrm{IL}-11$ may play an important role in the regulation of epithelial function under physiologic conditions and may exert potent anti-inflammatory effects during chronic intestinal inflammation. It is also possible that the inhibition of IL-11-modulated epithelial transport during an inflammatory insult may contribute to the pathophysiology of IBD.

\section{Materials and Methods}

\section{Animals}

The experiments were approved by the Animal Care and Use Committee at the Oklahoma City, Veterans Affairs Medical Center. Adult male Sprague Dawley rats were purchased from Charles River (Wilmington, Massachusetts). HLA-B27 transgenic rats, carrying high copy numbers of the human major histocompatibility class 1 allele B27 and $\beta_{2}$-microglobulin genes were obtained from Taconic (Germantown, New York). All animals were housed individually with a normal 12 hours light/dark cycle and provided with standard laboratory rat diet and water ad libitum.

\section{Experimental Design}

The experiments were designed to study the direct effect of rhIL-11 on the electrogenic ion transport in the jejunum and colon isolated from three groups of animals: 1) healthy control Sprague Dawley rats; 2) HLA-B27 transgenic rats with spontaneous chronic inflammation of the gastrointestinal tract; and 3) HLAB27 rats pretreated with rhlL-11 to heal the inflammation. In the third group, two different time periods of treatment were tested to establish the effects of rhlL-11 on inflammation and mucosal injury as well as on the compromised ability of rhlL-11 to cause changes in the electrogenic transport across the jejunal and colonic mucosa. The HLA-B27 rats received $33 \mu \mathrm{g} / \mathrm{kg}$ of rhlL-11 subcutaneously (sc) every other day during a period of either 1 week ( $3 \mathrm{sc}$ injections at 48-hour intervals) or 2 weeks (7 sc injections at 48-hour intervals). Stool consistency was evaluated as normal with formed stool pellets (score 0), soft with formed stool pellets (score 1), loose with no formation of stool pellets (score 2), or watery diarrhea (score 3). Scores were given before beginning rhlL-11 treatment and every other day during the treatment period. All animals were killed 3 hours after the last injection of
rhIL-11 and segments from the jejunum and colon were harvested for histologic analysis, MPO activity assay, and measurement of transmucosal electrogenic transport.

\section{Ussing Chamber Studies}

The isolated jejunum and colon were placed in icecold oxygenated Krebs bicarbonate buffer $(\mathrm{pH} 7.2)$ and intraluminal contents were removed by flushing the lumen with Krebs solution. Intestinal segments (approximately $2 \mathrm{~cm}$ in length) were carefully stripped of the external muscle layer and mounted in modified Ussing chambers (World Precision Instruments, Sarasota, Florida) with an opening surface area of $0.6 \mathrm{~cm}^{2}$. The Krebs buffer added to the serosal and luminal sides of the mucosal sheets contained $10 \mathrm{~mm}$ glucose or $10 \mathrm{~mm}$ mannitol, respectively (Perdue and Davison, 1986). Two pairs of agar-salt bridge electrodes connected to an EVC-4000 voltage/current clamp apparatus (World Precision Instruments) were used to monitor either the transmural PD under open-circuit conditions or the Isc when the transmural PD was clamped to zero. Under voltage-clamped conditions, changes in Isc are a measure of net active ion transport across the tissue. The PD or Isc were continuously recorded and measured using the MacLab Data Acquisition System (AD Instruments Ltd., Castle Hall, Australia). Individual values of basal PD (mV) or Isc $\left(\mu \mathrm{A} / \mathrm{cm}^{2}\right)$, and the maximal changes induced by drug treatment were measured using a customized data analysis option of the MacLab. Data were transferred to a Microsoft Excel spread sheet and subjected to "Descriptive Statistics" for calculating the mean, standard deviation, and standard error of the mean (SEM) for each group of experiments. Mean basal conductance of the mucosal tissue $\left(\mathrm{mS} / \mathrm{cm}^{2}\right)$ was calculated according to Ohm's law for each experimental group. After a 20 to 30 minutes equilibration period, cumulative concentrations of rhlL-11 ( 1 to $10,000 \mathrm{ng} / \mathrm{ml}$ final bath concentration) were added either to the serosal or luminal bathing solutions. Control experiments were carried out with vehicle $(0.1 \% \mathrm{BSA}$ in $0.9 \%$ saline $)$ or with a mutant rhlL-11 molecule that lacks biological activity.

\section{MPO Activity}

MPO, a granule-associated peroxidase primarily contained in polymorphonuclear neutrophils, is believed to be a specific enzymatic marker of neutrophil infiltration. Full-thickness tissue samples were isolated from the jejunum and colon and were immediately frozen in liquid nitrogen. The samples were stored at $-70^{\circ} \mathrm{C}$ and MPO activity was assayed simultaneously for each experimental group using a modification of the spectroscopic method of Castro et al (1974). The tissues were homogenized in $0.5 \%$ HTAB buffer $(\mathrm{pH} 6)$ at a 1:20 w/v ratio. On the day of the assay, extraction of MPO from the homogenate was performed by sonification on ice followed by three consecutive freezing-thawing procedures and centrifugation. MPO 
activity was tested in $10 \mu \mathrm{l}$ samples of the supernatant using a TMB Microwell Peroxidase Substrate System (Sigma Chemical Company, St. Louis, Missouri) and horseradish peroxidase as a relative standard. MPO activity was expressed as equivalent to the activity of the amount of horseradish peroxidase (ng) that converts the same amount of substrate in 10 minutes at room temperature.

\section{Histology}

Jejunal and colonic tissues were removed and standard regions of the jejunum $(5 \mathrm{~cm}$ distal to the ligament of Treiz) and a portion of the mid colon were examined as described previously (Keith et al, 1994; Peterson et al, 1998). Briefly, tissues were immersed in $10 \%$ neutral-buffered formalin, processed, paraffin embedded, sectioned at 5 microns, and stained with hematoxylin and eosin. Slides were scored for histologic lesions in a blinded fashion using the scale modified by Boughton-Smith et al (1988). Four parameters were taken into account by assigning 2 or 3 unit scales for each parameter: ulceration (0 to 2), inflammation (0 to 3), depth of lesion (0 to 3 ), and fibrosis (0 to 2). A score of 0 indicated the absence of pathology. The maximal total score per section was 10 .

\section{Drugs and Solutions}

All experiments were performed using a Krebs solution with $120 \mathrm{~mm} \mathrm{NaCl}, 6 \mathrm{~mm} \mathrm{KCl}, 2.5 \mathrm{~mm} \mathrm{CaCl}_{2}, 1.2$ $\mathrm{mm} \mathrm{NaH} \mathrm{PO}_{4}, 1.2 \mathrm{~mm} \mathrm{MgCL}$, and $14.4 \mathrm{~mm} \mathrm{NaHCO}_{3}$. The serosal bathing solution contained $11.5 \mathrm{~mm}$ glucose, whereas the mucosal bathing solution contained an equimolar concentration of mannitol. TTX (Sigma Chemical Company) was purchased as a mixture containing $1 \mathrm{mg}$ of TTX and $5 \mathrm{mg}$ of citrate buffer $(\mathrm{pH}$ 4.3) and dissolved in distilled water. A standard stock solution of the cytokine recombinant human IL-11 was kindly supplied by Genetics Institute, Inc., (Andover, Massachusetts). Serial dilutions were prepared in physiologic saline (0.9\%) consisting of $0.1 \%$ BSA (Sigma Chemical Company). The inactive rhlL-11 mutant control, supplied by Genetics Institute, Inc., was obtained by production of a mutant rhlL-11 molecule in which amino acid 58 was changed from methionine to alanine. This results in a 25 -fold decrease in receptor binding (Czupryn et al 1995). RhIL-11 was injected $\mathrm{sc}$ at a dose of $33 \mu \mathrm{g}$ in a volume of 0.1 to $0.2 \mathrm{ml}$.

\section{Expression of Results and Statistical Analysis}

All results are expressed as mean \pm SE. Standard statistical procedures were used to assess the significance of the effects and to compare data obtained in control and HLA-B27 rats. One-way analysis of variance was followed by a paired Student's $t$ test to compare data from tissues from the same animals or an unpaired Student's $t$ test to compare data between control and HLA-B27 transgenic animals. In each case, the level of statistical significance was set at $p<0.05$.

\section{Acknowledgements}

The authors would like to thank Dr. Kalina Venkova of the Oklahoma Foundation for Digestive Research Basic Science Labs for her assistance with analyzing the intestinal samples for MPO levels and her critique of the manuscript and Dr. Leo Albert from Genetics Institute for his assistance with the histology.

\section{References}

Alavi K, Prasad R, Lundgren K, and Schwartz MZ (2000). Interleukin 11 enhances small intestinal absorptive function and mucosal mass after intestinal adaptation. J Ped Sur 35(2):371-374.

Albert LM, Soley E, Ingram R, Warne N, Ferranti TJ, Erickson JE, Mason LE, Donnelly LH, Schaub RG, and Keith, JC Jr (1997). Luminal colonic treatment with rhlL-11 decreases bowel lesions in HLA-B27 rats. Gastroenterology 112:A920.

Asafa S, Bell CJ, Wallace JL, and MacNaughton WK (1999). Prolonged colonic epithelial hyporesponsiveness after colitis: Role of inducible nitric oxide synthase. Am J Physiol 276: G703-G710.

Baynes RD, Cook JD, and Keith J (1995). Interleukin-11 enhances gastrointestinal absorption of iron in rats. $\mathrm{Br} J$ Haematol 91:230-233.

Binder HJ, Foster ES, Budinger ME, and Hayslett JP (1987). Mechanism of electroneutral sodium chloride absorption in distal colon of the rat. Gastroenterology 93:449-455.

Boughton-Smith NK, Wallace JL, Morris GP, and Whittle BJ (1988). The effect of anti-inflammatory drugs on eicosanoid formation in a chronic model of inflammatory bowel disease in the rat. Br J Pharmacol 94:65-72.

Calkins BM and Mendeloff Al (1986). Epidemiology of inflammatory bowel disease. Epidemiol Rev 8:60-91.

Castagliuolo I, Kelly CP, Qiu BS, Nikulasson ST, LaMont JT, and Pothoulakis C (1997). IL-11 inhibits Clostridium difficile toxin A enterotoxicity in rat ileum. Am J Physiol 273:G333G341.

Castro GA, Roy SA, and Stockstill RD (1974). Trichinella spiralis: Peroxidase activity in isolated cells from the rat intestine. Exp Parasitol 36:307-315.

Chang EB, Field M, and Miller RJ (1982). Alpha 2-adrenergic receptor regulation of ion transport in rabbit ileum. Am J Physiol 242:G237-G242.

Chiossone DC, Simon PL, and Smith PL (1990). Interleukin-1: Effect on rabbit ileal mucosal ion transport in vitro. Eur J Pharmacol 180:217-228.

Czupryn MJ, McCoy JM, and Scoble HA (1995). Structurefunction relationships in human interleukin-11. Identifications of regions involved in activity by chemical modification and site-directed mutagenesis. J Biol Chem 270:978-985.

Du X, Liu Q, Yang Z, Orazi A, Rescorla FJ, Grosfeld JL, and Williams DA (1997). Protective effects of interleukin-11 in a murine model of ischemic bowel necrosis. Am J Physiol 272:G545-G552.

Greenwood B, Tremblay L, and Davison JS (1987). Sympathetic control of motility, fluid transport, and transmural PD in the rabbit ileum. Am J Physiol 16:G726-G729. 
Hammer RE, Maika SD, Richardson JA, Tang JP, and Taurog JD (1990). Spontaneous inflammatory disease in transgenic rats expressing HLA-B27 and human $\beta 2 \mathrm{~m}$ : An animal model of HLA-B27-associated human disorders. Cell 63:1099-1112.

Kao CY (1986). Structure-activity relationship of tetrodotoxin, saxitoxin, and analogues. Ann NY Acad Sci 479:52-67.

Keith JC, Albert A, Sonis ST, Pfeiffer CJ, and Schaub RG (1994). IL-11 a pleiotrophic cytokine: Exciting new effects of II-11 on gastrointestinal mucosal biology. Stem Cells 12:79-90.

Leng SX and Elias JA (1997). Interleukin 11. Int J Biochem Cell Biol 29:1059-1062.

Levin B (1992). Inflammatory bowel disease and colon cancer. Cancer 70 (Suppl 5): 1313-1316.

MacDonald TT and Spencer J (1988). Evidence that activated mucosal T-cells play a role in the pathogenesis of enteropathy in human small intestine. J Exp Med 167:1341-1349.

Madsen KL, Lewis SA, Tavernini MM, Hibbard J, and Fedorak RN (1997). Interleukin 10 prevents cytokine-induced disruption of T84 monolayer barrier integrity and limits chloride secretion. Gastroenterology 113:151-159.

Madsen KL, Tavernini MM, Mosmann TR, and Fedorak RN (1996). Interleukin 10 modulates ion transport in rats small intestine. Gastroenterology 111:936-944.

Orazi A, Du X, Yang Z, Kashai M, and Williams DA (1996). Interleukin 11 prevents apoptosis and accelerates recovery of small intestinal mucosa in mice treated with combined chemotherapy and radiation. Lab Invest 75:33-42.

Paul SR, Bennett F, Calvetti JA, Kelleher K, Wood CR, O'Hara RM, Leary AC, Sibley B, Clark SC, Williams DA, and Young YC (1990). Molecular cloning of cDNA encoding interleukin-11, a stromal cell-derived lymphopoietic and hematopoietic cytokine. Proc Natl Acad Sci U S A 87:7512-7516.

Perdue $\mathrm{MH}$ and Davison JS (1986). Response of jejunal mucosa to electrical transmural stimulation and two neurotoxins. Am J Physiol 251:G642-G648.
Peterson RL, Wang L, Albert L, Keith JC Jr, and Dorner AJ (1998). Molecular effects of recombinant human interleukin-11 in the HLA-B27 rat model of inflammatory bowel disease. Lab Invest 78:1503-1512.

Qiu BS, Pfeiffer CJ, and Keith JC (1996). Protection by recombinant human interleukin 11 against experimental TNB-induced colitis in rats. Dig Dis Sci 41:1625-1630.

Quesniaux VF, Mayer P, Liehl E, Turner K, Goldman SJ, and Fagg B (1993). Review of a novel hematopoietic cytokine, interleukin 11. Int. Rev of Exp Pathol 34A:205-214.

Sands BE, Bank S, Sninsky CA, Robinson M, Katz S, Singleton JW, Miner PB, Safdi MA, Galandiuk S, Hanauer SB, Varilek GW, Buchman AL, Rodgers VD, Salzberg B, Cai B, Loewy J, DeBruin MF, Rogge H, Shapiro M, and Schwertschlag US (1999). Preliminary evaluation of safety and activity of recombinant human interleukin 11 in patients with active Crohn's disease. Gastroenterology 117:58-64.

Sellin JH and Desoignie R (1984). Rabbit proximal colon: A distinct transport epithelium. Am J Physiol 246:G603-G610.

Sonis S, Muska J, O'Brian J, VanVugt A, Langer-Safer P, and Keith $J$ (1995). Alterations in the frequency, severity and duration of chemotherapy-induced mucositis in hamsters by interleukin-11. Eur J Cancer B Oral Oncol 31B: 261-266.

Targan SR, Deem RL, and Shanahan F (1991). Role of mucosal T-cell-generated cytokines in epithelial cell injury. Immunol Res 10:472-478.

Theodorou V, Eutamene H, Fioramonti J, Junien JL, and Bueno L (1994). Interleukin-1 induces a neurally mediated colonic secretion in rats: Involvement of mast cells and prostaglandins. Gastroenterology 106:1493-1500.

Zund G, Madara JL, Dzus AL, Awtrey CS, and Colgan SP (1996). Interleukin-4 and interleukin-13 differentially regulate epithelial chloride secretion. J Biol Chem 271:74607464. 